\title{
Optimal application of unmanned aerial vehicle in disaster relief
}

\author{
Tianying Xie ${ }^{1, \text { a }}$, Yantao $\mathrm{Li}^{2, \mathrm{~b}, *}$ \\ ${ }^{1}$ School of Computer and Information Science, Southwest University, Chongqing 400715, China \\ ${ }^{2}$ School of Computer and Information Science, Southwest University, Chongqing 400715, China \\ aastrmeteor@swu.edu.cn, byantaoli@swu.edu.cn
}

\begin{abstract}
Based on the theory of optimization, this paper researches the route planning of multi unmanned aerial vehicle(UAV) cooperative task and the task planning problem of multi UAV scheduling. In this paper, we set up a feature point location model based on the traditional base station location model, which is our basic model. The problem one researches the task planning of UAV cooperative investigation. We transform the problem into the multiple traveling salesman problem and use genetic algorithm to get the optimal number and route of UAV with high computational efficiency. The optimization model is also established, and the genetic algorithm is used to solve it, so as to calculate the optimal number and route of UAV and the flight time of each UAV. Problem two researches the multi-starting multi-unmanned aerial vehicle cooperative investigation task planning problem. Problem three researches the scheduling problem of UAV with communication constraints.
\end{abstract}

Keywords: UAV, Collaborative Planning, Genetic Algorithm, Nonlinear Optimization Model,

\section{Introduction}

\subsection{Background of the problem}

In August 8, 2017, a 7-magnitude earthquake occurred in Jiuzhaigou County, Aba state, Sichuan, causing irreparable casualties and major property losses. Because it is difficult to predict earthquake, timely and efficient disaster relief is an important measure to reduce the loss of earthquake. As a new vehicle, unmanned aerial vehicle (UAV) can play an important role in rescue operations. There are 2913 columns and 2775 lines of elevation data in the earthquake area.

\subsection{Symbolic description}

Table 1 Symbolic description

\begin{tabular}{|c|c|}
\hline Symbol & Description \\
\hline$\Omega=\{1,2,3, \cdots N\}$ & Feature point address set \\
\hline $\mathrm{K}=0$ & A region of $<2000$ meters above sea level \\
\hline $\mathrm{K}=1$ & $\begin{array}{l}\text { A region of 2000-3000 meters above sea } \\
\text { level }\end{array}$ \\
\hline$K=2$ & $\begin{array}{l}\text { A region of 3000-4000 meters above sea } \\
\text { level }\end{array}$ \\
\hline $\begin{array}{c}\mathrm{K}=3 \\
S^{l}\end{array}$ & $\begin{array}{c}\text { A region of }>4000 \text { meters above sea level } \\
\text { Area of region l }\end{array}$ \\
\hline$S_{i}^{l}$ & $\begin{array}{l}\text { The area of the unmanned aerial vehicle } \\
\text { (UAV) detection area covered with region l }\end{array}$ \\
\hline$r^{l}$ & Coverage requirements for regional l \\
\hline$x_{i j q}=\{0,1\}$ & $\begin{array}{c}\text { Reconnaissance situation of q-th unmanned } \\
\text { aerial vehicle (UAV) }\end{array}$ \\
\hline$t_{i j q}^{p}$ & $\begin{array}{l}\text { The flight time of q-th the unmanned aerial } \\
\text { vehicle in the p-th point of departure }\end{array}$ \\
\hline
\end{tabular}




\section{Problem analysis and model establishment solution}

\subsection{Problem one: disaster inspection}

\subsection{1 problem analysis}

The problem is a study of the task planning of the UAV. For the first small question, this problem can be simply expressed as: the best route and the UAV scheduling strategy are needed to make all UAVs longer than 3000 meters above sea level in the regional S. For second small queries, the minimum number of UAVs is still searching, so that within 72 hours, the time interval between the two places visited above is not greater than 3 hours.

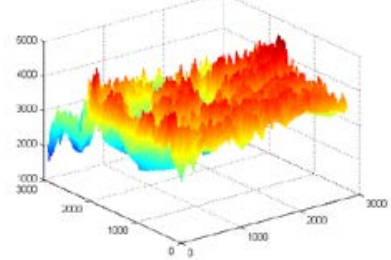

Figure 1 Target area 3D schematic diagram

Figure 1 is the elevation map of the whole area. Then, considering that when UAVs patrol the area $\mathrm{S}$ in the air, the observed range is different at different altitudes. Therefore, in order to facilitate the follow-up modeling and calculation, we divide the area into four grades at altitude, each of which is 1000 meters at intervals.

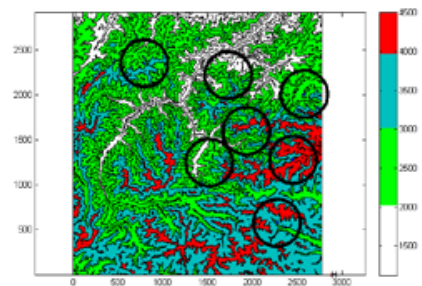

Figure 2 The elevation classification of the target area

\subsubsection{Model establishment}

$$
\begin{gathered}
\min F_{1}=|\Omega| \\
\text { s.t. } \sum_{i=1}^{n}\left(\frac{s_{k}^{i}}{S^{k}}\right)^{*}=r^{k}, k=\{0,1,2,3\}\left(x_{i}, y_{i}\right) \epsilon \\
\mathrm{U}=\sum_{j=1}^{m} U_{j} \\
U_{j}=\text { Peak }_{j} \times e^{\left(\left(x-\frac{h_{x j}}{h y_{r x j}}\right)^{2}+\left(\left(y-h_{y j}\right) / h_{r y j}\right)^{2}\right)}
\end{gathered}
$$

Formula 1 is the objective function of the model, representing the number of least feature points required to satisfy the constraint conditions, that is, the length of the set X. Formula 2 shows the coverage ratio of each unmanned aerial vehicle during its inspection.[1] Formula 3 said the UAV in this region is the mountain terrain threat, which $U$ said the threat caused by the peak area sum, m said the number of peaks in the region, $U_{j}$ said the j peaks of the threat, $P e a k_{j}$ is the j peak, $(\mathrm{x}, \mathrm{y})$ said the geographical coordinates of UAV, the gradient direction $\left(h_{x i}, h_{x j}\right)$.[2]

\subsubsection{Model solution}

Genetic algorithm solving model

The cross determines the global search ability of genetic algorithm, it also operates on two chromosomes, the characteristics of two new generations, the performance of genetic algorithm performance largely depends on the crossover operation, parental chromosomes are crossed by crossing rate control. The specific formulas are as follows:

$$
\begin{gathered}
Z_{L}(i)=\partial(i) P_{i}+(1+\partial(i)) P_{k}(i) \\
Z_{L+1}(i)=(1-\partial(i)) P_{i}+\partial(i) P_{k}(i)
\end{gathered}
$$

The basic algorithm flow is shown in Figure 3. 


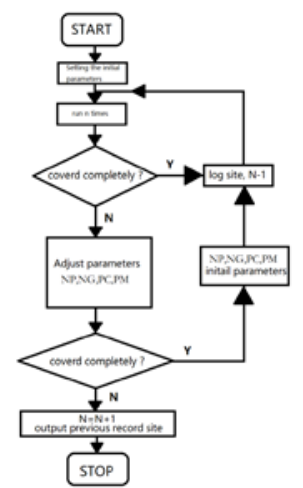

Figure 3 Flow chart of genetic algorithm

We set up the following model with the minimum number of unmanned aerial vehicles for investigation:

$$
\begin{gathered}
\min Q=\sum_{q} \sum_{i}^{N} \sum_{j}^{N}\left(x_{i j q} \cdot t_{i j q}\right) \\
\text { s. t. } \sum_{i}^{N} \sum_{j}^{N}\left(t_{i j} \cdot t_{i j q}\right) \leq 4
\end{gathered}
$$

The target function formula 7 represents the UAV that satisfies the constraints. [3] A schematic diagram of path planning, as shown in Figure 4.

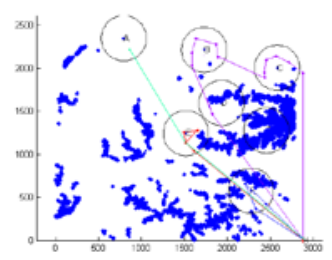

Figure 4 Schematic diagram of path planning

Genetic algorithms are used to solve multiple traveling salesman problem(MSTP) of m merchants and $n$ cities problem. The number of a feasible solution is $(n+m-1)$ !

Second question.

A. Model establishment

$$
\begin{gathered}
\operatorname{minQ}=\sum_{q} \sum_{i}^{N} \sum_{j}^{N}\left(x_{i j q} \cdot t_{i j q}\right) \\
\text { s.t. } \sum_{i}^{N} \sum_{j}^{N}\left(t_{i j} \cdot t_{i j q}\right) \leq 8 \\
\left|t_{q i_{1}}-t_{q i_{2}}\right| \leq 3 \\
\sum q \geq \frac{72}{t_{h}}
\end{gathered}
$$

Similarly, we use genetic algorithm to solve the above models. The first-round chromosome length 50. characteristic points are defined in the range of $0<x_{l}<106,0<y_{l}<110$. Finally, as shown in Figure 5, the number of UAVs is 45.

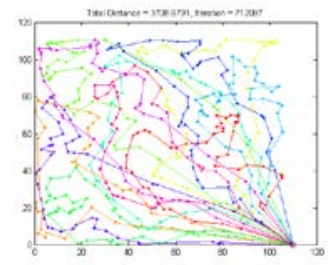

Figure 5 Patrol route under 4000 meters

\subsection{Problem two: detection of signs of life}

\subsection{1 problem analysis}

Problem 2 study the problem of multi-start multi-UAV cooperative investigation task planning. [4] It can be simply described as follows: Multiple UAVs go back to their starting points after investigating 
and completing tasks, and how to make UAVs detect more areas as possible as possible and complete the task for the shortest time.

\subsection{2 model establishment}

$$
\begin{gathered}
\operatorname{minT}=\sum_{p} \sum_{i=1}^{N} \sum_{j=1}^{N} \sum_{q=1}^{Q}\left(t_{i j q}^{p} \cdot x_{i j q}^{p}\right)+t_{0} \\
\text { s.t. } \sum_{i}^{N} \sum_{j}^{N}\left(t_{i j} \cdot t_{i j q}\right) \leq 8 \\
\sum_{i}^{N} x_{c}^{p}=1 \\
\sum_{q=1}^{Q} q=15, m=2
\end{gathered}
$$

\subsubsection{Model solution}

We use genetic algorithm to optimize the solution, and the process is similar to the solution process in the 2.1.3. We run the code by MATLAB, and we get the obtained coordinates. The flight route of the UAV can be obtained through the obtained coordinates, such as Figure 6 .

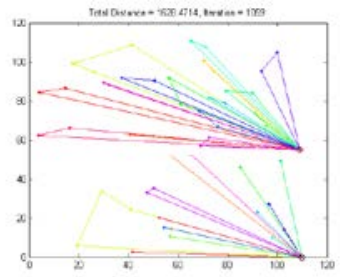

Figure 6 route map of unmanned aerial vehicle from base

\subsection{Problem three: Communication relay in disaster area}

\subsection{1 problem analysis}

It is considered that the mobile terminal on the ground is stationary. We solve this problem by establishing a hierarchical programming model. As shown in Figure 7.

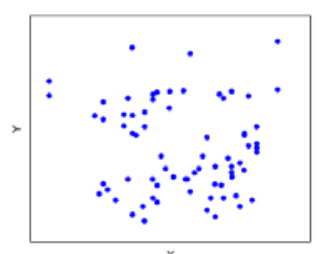

Figure 7 ground terminal geographic location

According to the position of the above ground terminal, we use the clustering method to analyze it. First we use MATLAB to write K-mean clustering algorithms.[5] As shown in Figure 8.

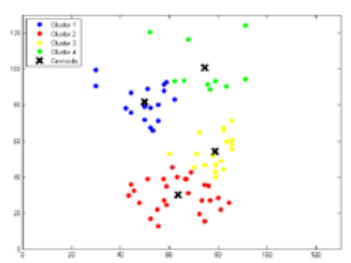

Figure 8 K-mean clustering

The model will get four kinds of regions, then transform the problem into at least four UAVs in each of the four regions.

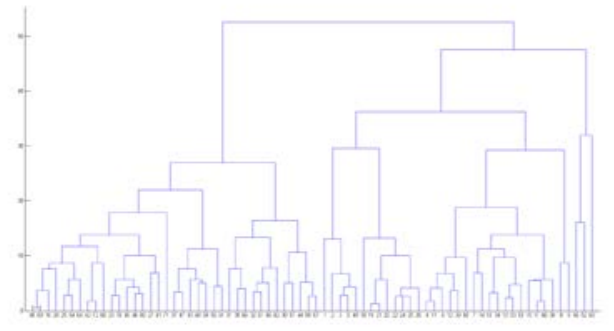

Figure 9 Cluster tree graph 


\subsubsection{Model establishment and solution}

$$
\begin{gathered}
\operatorname{minF}=|\Omega| \\
\text { s.t. } \sum_{i=1}^{n}\left(\frac{s_{i}^{k}}{s^{k}}\right)^{*}=r^{k} \\
\left(x_{i}, y_{i}\right) \in \mathrm{D} \\
\min F_{1}=\sum_{i=1}^{n} x_{i} \\
\text { s.t. } \sum_{i=1}^{n}\left(\frac{s_{i}^{k}}{s^{k}}\right)^{*}=r^{k}, k=\{0,1,2,3\} \\
\left\|d_{x_{i}}-d_{x_{j}}\right\|<3 \mathrm{~km} \\
\min F_{1}=\sum_{i=1}^{n} x_{i} \\
\text { s.t. } \sum_{i=1}^{n}\left(\frac{s_{i}^{k}}{s^{k}}\right)^{*}=r^{k}, k=\{0,1,2,3\} \\
\left\|d_{x_{i}}-d_{x_{j}}\right\|<6 \mathrm{~km}
\end{gathered}
$$

Communication relay planning diagram of UAV ass shown in Figure 10.

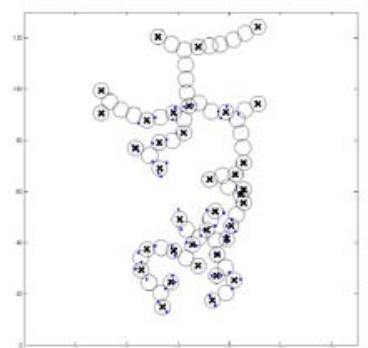

Figure 10 Communication relay planning diagram of UAV

\section{Conclusions}

In this paper, according to theory of optimization, We proposed a novel feature point location model based on the traditional base station location model. Our model can clearly enhance the application of disaster relief via UAV. We choose the feature point address selection model and select the feature point address in each key area of the target area, so that we can transform the complex route planning to solve the shortest path to the S point. Based on the theory of optimization, we obtained the route planning of UAV cooperative task and the task planning problem of multi UAV scheduling.

\section{Acknowledgments}

I'm deeply indebted to a number of people without whose encouragement and assistance this thesis would not have been completed.

\section{References}

[1] Shang K, Karungaru S, Feng Z, et al. Periodic re-optimization based dynamic branch and price algorithm for dynamic multi-UAV path planning[C]// IEEE International Conference on Mechatronics and Automation. IEEE, 2013:581-586.

[2] Márcio da Silva Arantes, Arantes J D S, Toledo C F M, et al. A Hybrid Multi-Population Genetic Algorithm for UAV Path Planning[C]// Genetic and Evolutionary Computation Conference. ACM, 2016:853-860.

[3] Madraswala H S, Deshpande A S. Genetic algorithm solution to unit commitment problem[C]// International Conference on Nascent Technologies in Engineering. IEEE, 2017:1-6.

[4] Asadi E, Silva M G D, Antunes C H, et al. A multi-objective optimization model for building retrofit strategies using TRNSYS simulations, GenOpt and MATLAB[J]. Building \& Environment, 2012, 56(10):370-378.

[5] Selim S Z. K-mean-type algorithms[J]. IEEE Trans.pattern Anal. \& Mach.intell, 1984, 6. 